\title{
Die Reise der Dominikanerinnen von Silo nach Kirchheim unter Teck 1478 im Kontext der spätmittelalterlichen Klosterreform
}

\author{
Von Stefanie Monika Neidhardt
}

\section{Einleitung}

Die Reise der observanten Dominikanerinnen im Jahr 1478 von Silo im Elsass, dem heutigen Sélestat, in das Kloster Kirchheim unter Teck ist der Chronistin Magdalena Kremer einen längeren Eintrag in ihre Kirchheimer Chronik wert ${ }^{1}$. Die Autorin, die von sich selbst bescheiden in der dritten Person spricht und sagt: ein swester... die konde wol textur schriben und ouch molen ${ }^{2}$, war als Novizenmeisterin, Vorsängerin und Meßnerin wegen ihrer besonderen Fähigkeiten für das Kirchheimer Kloster ausgewählt worden.

Nach ihrer Beschreibung reisten die observanten Dominikanerinnen aus dem Kloster Silo über St. Margarethen in Straßburg nach Lichtenau und Ettlingen. Pfingsten verbrachten sie im bereits reformierten Dominikanerinnenkloster Pforzheim und zogen dann Richtung Stuttgart weiter. Nach kurzem Aufenthalt kamen

${ }^{1}$ Vgl. den bislang einschlägigen Druck bei Christian Friedrich Sattler: Geschichte des Herzogthums Würtenberg unter der Regierung der Graven. Bd.4. Tübingen 1768. Beilage 42, S. 173-296 (von nun an zitiert als Kirchheimer Chronik). Hier S. 155-156. Die Passage des Reiseberichts in der Kirchheimer Chronik wird hier im Anhang nach der Vorlage im Hauptstaatsarchiv Stuttgart neu ediert und entsprechend zitiert (HStA Stuttgart A 493 Bü 2 S. 10-13). - Vgl. dazu Ulrich P. Ecker: Die Geschichte des Klosters S. Johannes-Baptista der Dominikanerinnen zu Kirchheim u. Teck. Freiburg 1985. - Zu Kloster Kirchheim und Magdalena Kremer: Rolf Götz: Die älteste Urkunde des Kirchheimer Frauenklosters. In: Schriftenreihe des Stadtarchivs Kirchheim unter Teck 3 (1985) S.7-23. - Roland Deigendesch: Die „Frau von Mantua“ und das Dominikanerinnenkloster in Kirchheim unter Teck. In: Von Mantua nach Württemberg: Barbara Gonzaga und ihr Hof. Begleitbuch und Katalog zur Ausstellung des Landesarchivs Baden-Württemberg, Hauptstaatsarchiv Stuttgart. Bearb. von Peter Rückert. Stuttgart ${ }^{2} 2012$. S. 161-167. - Jeffrey F. Hamburger: Magdalena Kremerin, Scribe and Painter of the Choir and Chapter Books of the Dominican Convent of St. Johannes-Baptista in Kirchheim unter Teck. In: The Medieval Book. Glosses from Friends and Collegues of Christopher de Hamal. Hg. von James H. Marrow. Houten 2010. S. 124-149. Sönke Lorenz: Art. ,Kremer, Magdalena‘. In: Verfasserlexikon 11 (2004) Sp. 892-893.

${ }^{2} \mathrm{Vgl}$. Anhang. 
die Schwestern in dem ebenfalls zu reformierenden Kloster in Weiler bei Esslingen an und erreichten dann ihr eigentliches Ziel, das Kloster Kirchheim unter Teck, das unter ihrer Mithilfe ein Reformkloster werden sollte, und für dessen Ämter die Schwestern aus Silo vorgesehen waren.

Jede Reise war für die Schwestern, die die Stabilitas Loci und die Klausur auf sich genommen hatten, ein außergewöhnliches Abenteuer, der Wechsel in einen zu reformierenden fremden Konvent und das weitere Leben dort eine große und riskante Angelegenheit. Diese Fahrt ins Ungewisse war weder Selbstzweck noch Vergnügen, sondern diente dem einen Ziel, die Reform und damit den idealen Weg zu Gott zu verbreiten. Ereignisse und Reiseweg sind für die Chronistin und spätere Vorsängerin nicht nur wichtig, um diesen Vorgang in die Reformen des Dominikanerordens im 15. Jahrhundert einzuordnen, sondern auch um die Vernetzung im Orden darzustellen und Verbindungen zu anderen Reformklöstern ${ }^{3}$ zu begründen. Auch bezeugt Magdalena Kremer die Fürsorge des zukünftigen Landesherren Graf Ulrich V. von Württemberg, der die Schwestern auf der Reise in ihrer strengen Einhaltung der Regel bestärkte und diese so angenehm wie möglich machte.

Forschungen über das Reisen im Mittelalter und in der frühen Neuzeit stehen zur Zeit im Fokus des historischen Interesses. Fragestellungen zu Anlässen und Motiven des Reisens, Fremdwahrnehmung, Kulturkontakt, Wirkungen und Spu$\mathrm{ren}^{4}$ spielen auch in diesem besonderen Kontext der Reise der Siloer Schwestern im Folgenden ebenfalls eine Rolle. Beeinflusst von Schönensteinbach bei Gebweiler, das 1397 als Reformkloster gegründet worden war, Unterlinden bei Colmar (gegründet 1419) und St. Nikolaus in Undis in Straßburg (gegründet 1431) wurde Silo in der Reichsstadt Schlettstadt 1463 reformiert $^{5}$. Es zeugt von der Kraft der Reformbewegung und vom Wohlstand dieses Klosters, dass es schon relativ kurze Zeit nach der Reform sieben Schwestern für leitende Aufgaben abgeben und dotieren konnte ${ }^{6}$.

Die Schwestern begaben sich auf die Reise von Silo nach Kirchheim, um am Ende ihrer Reise anderen Schwestern die dominikanische Observanz als ein festes Ziel und Ideal des Lebens auf Gott hin nahezubringen. Neben diesem hohen Ziel

${ }^{3}$ Die Kirchheimer Chronik berichtet dazu späterhin, dass die Äbtissin in höchster Not während der Belagerung durch Eberhard den Jüngeren andere Klöster, darunter auch Pforzheim, in einem Manifest um Hilfe bat. Kirchheimer Chronik (wie Anm. 1) S. 206.

${ }^{4}$ Vgl. dazu den aktuellen Forschungsstand bei Folker Reichert und Peter Rückert: Reisen und Reiseliteratur im Spätmittelalter und in der frühen Neuzeit. In: ZWLG 68 (2009) S. 1119. - Daneben auch Klaus Herbers: Pilgertraditionen und Jakobsspuren in Südwestdeutschland. In: Ebd. S. 19-41. - Stefan Schröder: Dess glich ich all min tag ie gesechen hab und ob got will nùt mer sechen will. Fremd- und Selbstbilder in den Pilgerberichten des Ulmer Dominikaners Felix Fabri. In: Ebd. S. 41-64.

5 Angelus Walz: Dominikaner und Dominikanerinnen in Süddeutschland (1225-1966). Freising 1967. S. 50-51.

${ }^{6}$ Kirchheimer Chronik (wie Anm. 1) S. 176, 178. 
erhält die Reise ihre besondere Bedeutung im Kontext ihrer Organisatoren und Auftraggeber, den geistlichen und weltlichen Förderern der Reform.

\section{Die dominikanische Klosterreform im deutschen Südwesten}

Die Reform des Dominikanerordens hatte um die Mitte des 15. Jahrhunderts Süddeutschland erreicht, ausgelöst durch das 1390 in Italien verfasste Dekret des Ordensgenerals Raymund von Capua. Dieser forderte eine Rückbesinnung auf die Werte und Ideale des Heiligen Dominikus einhergehend mit der strengen Einhaltung der Ordensregel, was sowohl Zustimmung als auch heftige Proteste im Orden zur Folge hatte ${ }^{7}$. Eine strengere Beachtung der Ordensregeln, Klausur, Keuschheit, persönlichen Armut und eine Erneuerung der Liturgie waren die Kernpunkte dieser Reform. Außerdem legte man großen Wert auf Frömmigkeit, die sich darin ausdrückte, dass man die Stundengebete einhielt, das Fasten, die Lesungen, die Zeiten des Schweigens und die gemeinschaftlichen und persönlichen Gebete wieder einführte. Ein Rückzug aus der Welt und eine Vorliebe für individuelles Gebet und Meditation sollten zu einer Imitatio Christi führen ${ }^{8}$.

35 Jahre später setzte sich der Ordensbruder Johannes Nider literarisch mit den Widerständen gegen die sogenannte Observanz auseinander und widerlegte die Vorwürfe, die neue strengere Lebensweise sei wider die Natur und bringe nur Unfrieden unter den Ordensmitgliedern. Für ihn wurde die Observanz das „Salz für die Kirche“ und der Orden die Truppe Gottes, die durch Regeln und Vorschriften geordnet sein müsse, um Gott dienen zu können?.

In der Nachfolge Niders reformierte Johannes Meyer im süddeutschen Raum maßgeblich den Dominikanerorden. Unter seinen ersten reformierten Konventen waren die Schwesternkonvente Adelshausen, St. Agnes und St. Maria Magdalena in Freiburg $^{10}$. Später gewann Meyer auch Einfluss auf den elsässischen Raum. Ihm verdanken wir mit der Chronik des Klosters Schönensteinbach eine ausführliche Beschreibung der Vorgehensweise und des Voranschreitens der observanten Reform $^{11}$. Die Dominikanerinnen als seine Leserinnen sollten durch das Wissen um

${ }^{7} \mathrm{Vgl}$. Eugen Hillenbrand: Die Observantenbewegung in der deutschen Ordensprovinz der Dominikaner. In: Reformbemühungen und Observanzbestrebungen im spätmittelalterlichen Ordenswesen (Berliner Historische Studien 14). Hg. von Kaspar Elm. Berlin 1989. S. 219-271. Hier: S. 220.

${ }^{8}$ Vgl. Bernhard Neidiger: Standesgemäß leben oder frommes Gebet? Die Haltung der weltlichen Gewalt zur Reform von Frauenklöstern im 15. Jahrhundert. In: Rottenburger Jahrbuch für Kirchengeschichte 22 (2003) S. 201-220. Hier: S. 202.

9 Vgl. Hillenbrand (wie Anm. 7) S. 223.

10 Vgl. Franz Egger: Die Reform des Baseler Konvents 1429 und die Stellung des Ordens am Baseler Konzil 1431-1448. Bern u.a. 1991. S. 26-27.

11 Vgl. Johannes Meyer: Buch der Reformacio des Predigerordens. IV. und V. Buch. Hg. von Benedictus Maria Reichert. Leipzig 1908. S. 97-100. - Zur Dominikanerinnenreform im 
die leidvolle Reformarbeit und die Ideen des sittlichen Lebens selber zu einer tugendreichen Lebenseinstellung geführt werden. Seine Schriften galten als ein Ansporn zur Nachahmung und Stabilisierung des Lebens im observanten Kloster und zur Vorbereitung des Anschlusses an die Observanz.

In seinem Buch über die Reformbewegung zeigte Johannes Meyer anhand des Schwesternkonvents Schönensteinbach, wie eine gelungene Reform nach seiner Vorstellung auszusehen hatte. Als Ergebnis wird die Reform dadurch gerechtfertigt, dass neue Menschen in die Klöster einzögen und sie bevölkerten. Man muss mit Meyer daraus ableiten, dass die Reform der Frömmigkeit weiteren Kreisen entgegenkam und entsprach. Seinen Lesern und Leserinnen wollte Meyer eindeutig die Ideale der Reform vor Augen führen und sie dazu auffordern, nach der ursprünglichen Regel des Heiligen Dominikus zu leben. Während die Nonnen möglichst innerhalb der Klausur bleiben sollten, sah Meyer für die Mönche des Dominikanerordens vor, hinauszugehen und zu predigen, Schriften zu verfassen und für die Observanz zu werben. Für die Frauen hingegen sei die höchste Form der Gelehrsamkeit erreicht, wenn sie die Bibel auf Latein verstünden ${ }^{12}$. Er schlug für Nonnen eine Bücherliste und einen Leseplan vor, um sie im Sinne der Reform zu bilden. Dies hatte erhebliche Auswirkungen auf Bibliotheken und Skriptorien der Klöster. Nach Meyer sollten beide Ordensstränge sich ergänzen und voneinander lernen, um die Reformbewegung vollständig durchzuführen, was durch Visitation der Dominikanerprovinziale in den Frauenklöstern und durch die Beichtväter ermöglicht wurde, die eine wichtige Rolle für die Gewissensbildung und die Wissensvermittlung bei den Nonnen spielten.

Die Reform kam der zeitgenössischen Frömmigkeit und der Sehnsucht nach einem idealen monastischen Leben entgegen und schlug eine Brücke aus der Zeit der Ordensgründung in die zweite Hälfte des 15. Jahrhunderts. Insgesamt wurden mit ihr eine Intensivierung und Konzentration auf die Frömmigkeit und auf das Gebet, größere Schlichtheit des Gottesdienstes und des Lebens im Kloster erreicht, sowie die Rückbesinnung der Priorin auf ihre geistlichen Aufgaben, die Eindämmung von Adelsprivilegien, ein neues Interesse an den Studien, eine wirtschaftliche Erholung und Absicherung der Klöster ${ }^{13}$.

Elsass, besonders in Straßburg, sowie Johannes Meyer vgl. jetzt auch Sigrid Hirbodian: Dominikanerinnenreform und Familienpolitik. Die Einführung der Observanz im Kontext städtischer Sozialgeschichte. In: Schreiben und Lesen in der Stadt. Literaturbetrieb im spätmittelalterlichen Straßburg (Kulturtopographie des alemannischen Raums 4). Hg. von Stephen Mossman, Nigel F. Palmer und Felix Heinzer. Berlin/Boston 2012. S. 1-16.

12 Vgl. Heike Uffmann: Wie in einem Rosengarten. Monastische Reformen des späten Mittelalters in den Vorstellungen von Klosterfrauen. Bielefeld 2008. S. 72-76.

13 Vgl. Petrus Becker: Erstrebte und erreichte Ziele benediktinischer Reformen im Spätmittelalter. In: Reformbemühungen und Observanzbestrebungen im spätmittelalterlichen Ordenswesen (Berliner Historische Studien 14). Hg. von Kaspar Elm. Berlin 1989. S.23-34. Hier: S. 33-34. 
Die Reformbewegung musste nicht nur von geistlicher, sondern auch von weltlicher Seite getragen werden, um Erfolg zu haben. Die weltliche Herrschaft war an der Reform interessiert, da sie ihren Einfluss auf das zu reformierende Kloster auch hinsichtlich des zuverlässigen Totengedenkens ihrer Familie stärken konnte. Mit einem Kloster, das über gebildete Mönche oder Nonnen verfügte, erhoffte sich der Landesherr, in seinem Herrschaftsgebiet ein geistiges Zentrum zu schaffen, möglichst ohne sozialen Wettbewerb und mangelnde wirtschaftliche Absicherung wie an den Universitäten. Die reformierten Dominikanerklöster bemühten sich innerhalb eines geregelten Zusammenlebens um Wissenschaft und die Erneuerung der Liturgie, besaßen ihre eigene Frömmigkeit, und man konnte ihnen das Gedenken und Gebet für die verstorbenen Familienangehörigen unbesorgt anvertrauen ${ }^{14}$, $\mathrm{da}$ sie Zentren der Frömmigkeit und Orte des Friedens, der Ruhe und des Begräbnisses waren.

\section{Die Kirchheimer Chronik als Dokument der Klosterreform}

Die Kirchheimer Chronik kann als außergewöhnliches Dokument der spätmittelalterlichen Klosterreform gelesen werden. Sie wurde offensichtlich von der Dominikanerin Magdalena Kremer wohl um 1489/90 geschrieben ${ }^{15}$. Die Chronistin, gebürtig aus Straßburg ${ }^{16}$, war 1478 vom Schlettstädter Kloster Silo im Zuge der Reform nach Kirchheim gekommen und folglich Augenzeugin der Reise und der Reform. Ihre Chronik, wovon sich eine zeitnahe Abschrift heute im Wiener Schottenstift befindet ${ }^{17}$, wurde von Christian Friedrich Sattler bereits 1777 in seinem umfassenden Werk zur „Geschichte des Herzogthums Würtenberg unter der Regierung der Graven“ ediert ${ }^{18}$. Die Handschrift war nach der endgültigen Aufhebung des Klosters Kirchheim 1567 und dem Umzug der drei verbliebenen Schwestern ins Kloster Kirchberg nicht wie andere Klosterdokumente ins Kloster St. Blasien gelangt. Im 17. Jahrhundert taucht sie zunächst im Privatbesitz des evangelischen Dekans, herzoglichen Rats und Abts von Lorch Johann Wendelin Bilfin$\operatorname{ger}^{19}$ (1647-1722), dann in dem des Magisters Christoph Jakob Klupffel ${ }^{20}$ (1683-

${ }^{14}$ Dieses Gedenken wurde einigen Frauenklöstern ganz explizit übertragen, wie etwa den Nonnen des Klosters Schönensteinbach. Vgl. dazu Felix Fabri: Tractatus de civitate Ulmensi. Edition und Übersetzung (Bibliotheca Suevica 35). Hg. von Folker Reichert. Konstanz 2012. S. 303. - Vgl. dazu auch Hillenbrand (wie Anm. 7). S. 254.

15 Vgl. dazu den in Anm. 1 zitierten Forschungsstand.

16 Vgl. Kirchheimer Chronik (wie Anm. 1) S. 179. - Ecker (wie Anm. 1) S. 104.

17 Vgl. Uffmann (wie Anm. 12) S. 330.

18 Kirchheimer Chronik (wie Anm. 1) S. 173-296.

${ }^{19}$ Christian Sigel: Das evangelische Württemberg. Seine Kirchenstellen und Geistlichen von der Reformation bis auf die Gegenwart. Gebersheim 1910-1932. S. 337.

20 Siehe dazu die Einträge auf der ersten Seite der Kirchheimer Chronik in chronologischer Anordnung (wie Anm.1): 1621 (gestrichen). Darüber: $M$ [agister] Wendelini Bilffin- 
1740), Pfarrer in Ensingen, wieder auf und ging anschließend in den Besitz des Archivars und Geschichtsschreibers Christian Friedrich Sattler über.

Programmatisch beschäftigt sich die Kirchheimer Chronik mit der Einführung der Observanz im Kloster, deren Ziel es war, durch strengere Einhaltung der Regel die Seelen der Schwestern für Christus rein zu bewahren ${ }^{21}$. Sie schließt dabei den Konflikt mit dem neuen Landesherren, Graf Eberhard dem Jüngeren von Württemberg, an, der die Reform des Klosters rückgängig machen wollte. Der Durchhaltewillen der Schwestern von Silo als Anführerinnen des übrigen Konvents trotzte Aushungerung, Belagerung und der Androhung der Erstürmung des Klosters. Vor diesem Hintergrund beschreibt die Autorin die Reform von Kirchheim gleichzeitig als beeindruckendes Glaubenszeugnis ihrer Mitschwestern ${ }^{22}$. Magdalena Kremer ordnet dabei nicht nur die Reform des Einzelklosters in den Kontext der Observanzbewegung ein, sondern gibt auch Auskunft über das Leben der Schwestern im Kloster.

Solche Klosterchroniken, die für die Observanzbewegung geschrieben wurden, ermöglichten es den Schwestern auch, ein Netzwerk zum Austausch von Literatur zwischen den reformierten Klöstern zu schaffen. Auf diese Weise konnten sie eine aktive Rolle im spätmittelalterlichen Dialog um die Observanzbewegung einnehmen $^{23}$. Freilich wurden die Chroniken zunächst zur Belehrung der derzeitigen und

gerisum (gestrichen). Rechts darunter: Dono dedit Vidua Domini consulis Bülffingeri b [eate] $m$ [emorie] $M$ [agistro] Christophoro Jacobo Klupffelio, Pastori Ensing[ensis] infer[ioris] 1730. Links daneben: Filius Domini Klupfelii dono reliquit Christiano Friderico Sattlero Archivario Wirtenb [ergico] 1742. Oben: Christianus Fridericus Sattler Archivo Ducali donat in hui[us] memoriam die 1. Nov[embris] 1771.

Die Chronik wurde von Wendlin Bilfingers Witwe 1730 an Jakob Kluppfel übergeben. Dessen Sohn übergab sie 1742, zwei Jahre nach seinem Tod, an Christian Sattler. Johannes Wendlin Bilfinger durchlief sein Diakonat in Neuffen, Urach, St. Leonard in Stuttgart, bevor er Dekan und Pfarrer in Cannstatt und 1707 Abt des Klosters Lorch wurde.

Christoph Jakob Klupffel/ Klüpfel wurde am 28.6. 1683 in Stuttgart geboren und studierte im Stift in Tübingen. Nach einer Stelle als Pfarrer in Hattenhofen ab 1706 gelangte er 1722 nach Unterensingen. Ab 1736 war er Stiftspfarrer und Hofprediger in Langenburg. Vgl. Sigel (wie Anm. 19) S. 288. - Gerhard Hergenröder: Unterensingen. Geschichte einer Gemeinde. Unterensingen 1995. S. 61.

${ }^{21}$ Vgl. Thomas Lentes: Bild, Reform und Cura Monialium. Bildverständnis und Bildgebrauch im Buch der Reformacio Predigerordens des Johannes Meyer ( $\dagger 1485)$. In: Jean Charles Winnlen (Hg.) Dominicains et Dominicaines en Alsace XIII ${ }^{e}-X X^{\mathrm{e}}$ Siècle. Colmar 1996. S. 177-195.

22 Siehe Anm. 1.

${ }^{23}$ Vgl. Anne Winston-Allen: Convent Chronicles. Women Writing about Women and Reform in the Late Middle ages. Pennsylvania 2004. S. 5. - Dazu auch dies.: Es ist nit zu gelobind, daz ain frowen bild so wol kann arbaiten: Women's Accounts of Artistic Production and Exchange in Convents of the Observant Reform. In: Frauen - Kloster - Kunst. Neue Forschungen zur Kulturgeschichte des Mittelalters. Hg. von Jeffrey F. Hamburger u.a. Turnhout 2007. S.187-195. - Dies.: Rewriting Women's History: Johannes Meyer and the Fifteenth-Century Sister-Book. In: Medieval Voices in the $21^{\text {st }}$ Century. Hg. von Albrecht Classen. Amsterdam 1999. S. 145-154. 
künftigen Bewohnerinnen des Klosters geschrieben, wobei es allerdings die Perspektive der erfolgreichen - weil reformierten - Seite zu beachten gilt ${ }^{24}$.

Die Kirchheimer Chronik setzt mit dem Jahr 1476 ein, dem Beginn der Reform der Dominikanerinnenklöster in der Grafschaft Württemberg durch Graf Ulrich. Mit dem Hinweis auf jahrelange Verhandlungen um die Reform innerhalb des Ordens beweist die Chronistin ihre Kenntnisse von den inneren Abläufen im Dominikanerorden. Zum Gelingen einer Reform mussten Graf Ulrich von Württemberg und der Ordensobere Leonard von Perus langwierige Verhandlungen, auch über die hohen Kosten, führen. Die Grundlage ihres Berichts über diese Phase war wohl der Briefwechsel der Ordensoberen mit dem Grafen, den Magdalena Kremer bei der Abfassung ihrer Chronik in Kirchheim vorliegen hatte. Der Ordensobere schrieb an den Provinzial Jakob von Stubach, dass er „im heiligen Gehorsam und in der Kraft des Heiligen Geistes “25 die Reform durchführen solle.

Die Initiative und der Impuls zur Reform gingen von den Grafen von Württemberg aus, die sich das Recht zu dieser Reform von Papst Pius II. ${ }^{26}$ in Rom hatten verleihen lassen ${ }^{27}$. So beschreibt Magdalena Kremer den Ablauf der Reform in ihren einzelnen Etappen, ausgehend vom Recht der württembergischen Grafen aufgrund einer päpstlichen Erlaubnis zur Klosterreform über die Überzeugungsarbeit bei den Oberen des männlichen Ordenszweiges bis zur Bitte um reformierte Nonnen aus Schlettstadt. Die Chronistin weist auf sorgfältige Planung hin und nennt die wichtigsten Träger des Reformgedankens wie Leonard von Perus, Jakob von Stubach, Johannes Pruser und Johannes Meyer. Das Ansehen der Letzteren gilt der Autorin wegen der Erfolge bei der Reform in den genannten Klöstern in Freiburg im Breisgau als Garantie für eine gute Grundlage für die Reform in Württemberg ${ }^{28}$.

Im Anschluss beschreibt Magdalena Kremer die Vorbereitung der Nonnen aus Kloster Silo in Schlettstadt für die Reise nach Kirchheim, lässt dabei aber die eigentliche Auswahl der Nonnen für die neuen Ämter im Kloster durch die Priorin und die observanten Ordensreformer aus. Es ist anzunehmen, dass die Wahl nach einem bekannten Procedere ablief, von dem die Autorin nur Gebete zum Heiligen Geist, der Mutter Gottes und andere Gebete nennt ${ }^{29}$. Die eigentliche Vorgabe zur

${ }^{24}$ Vgl. Winston-Allen, Convent Chronicles (wie Anm. 23) S. 201.

${ }^{25}$ Kirchheimer Chronik (wie Anm. 1) S. 174.

${ }^{26}$ Bernhard Neidiger: Papst Pius II. und die Klosterreform in Deutschland. Eine Problemskizze. In: Vita Religiosa im Mittelalter. Festschrift für Kaspar Elm zum 70. Geburtstag. Hg. von Franz Felten und Nikolas Jaspert. Berlin 1999. S. 628-652.

${ }^{27}$ Vgl. Thomas Fritz: Ulrich V., der Vielgeliebte. In: Das Haus Württemberg. Ein biographisches Lexikon. Hg. von Sönke Lorenz u.a. Stuttgart u.a. 1997. S. 86-89. Hier: S. 88. Thomas Fritz: Ulrich der Vielgeliebte (1441-1480). Ein Württemberger im Herbst des Mittelalters. Zur Geschichte der württembergischen Politik im Spannungsfeld zwischen Hausmacht, Region und Reich (Schriften zur südwestdeutschen Landeskunde 25). Leinfelden-Echterdingen 1999.

${ }^{28}$ Vgl. Kirchheimer Chronik (wie Anm. 1) S. 174.

29 Vgl. Ebd. 
Wahl, die die Ratsschwestern auf Befehl des Provinzials ausführten, war, dass das Kirchheimer Kloster mit den wichtigsten Ämtern versorgt werden sollte und Silo nicht allzu sehr geschädigt wurde. Die Ratsschwestern wählten die abgeordneten Schwestern für ein Amt im neuen Kloster aus, weil bei einer Reform eine Besetzung der wichtigen Ämter mit reformierten Nonnen dringend nötig war. Es fand keine freie Wahl unter den Schwestern statt, sondern die Schwestern wurden gezielt nach ihren Fähigkeiten ausgesucht, um der Observanz ideal zu nützen. Auch kann man davon ausgehen, dass es sich bei den Schwestern um erfahrene, ältere Schwestern handelte; nach Winston-Allens Schätzung waren die zukünftige Äbtissin Barbara Bernheimer zur Zeit ihrer Wahl älter als 50 und die Subpriorin Elisabeth Herwert bereits $67 \mathrm{Jahre} \mathrm{alt}^{30}$.

\section{Die Vorbereitung der Reise}

Der Weggang von Silo in die Fremde fiel weder den Schwestern noch ihrem Beichtvater leicht, für sie waren das neue Kloster und die neue Umgebung fremd, die Fahrt führte ins Ausland, in das ellend ${ }^{31}$. Die Schwestern erhielten angesichts ihres Aufbruchs ins Ungewisse im Kloster noch das beylig sacrament ${ }^{32}$ : ... Und also furent die vii swestern von $S y l u \beta$ uff Johannis ante portam latinam am morgen vor tag, und gab jnen ir bychtvatter zu Syl das heylig sacrament zületz und in das ellend zui eynem geworen geleyt. ${ }^{33}$

Dadurch waren die Schwestern nicht nur im Stand der Gnade, sondern auch gestärkt gegen Unfälle, Versuchungen und Krankheit auf der Reise, Gott war mit ihnen und sollte durch seine Gnade das Gelingen ihrer Absicht garantieren. Der Empfang der Kommunion war auch durch den besonderen Anlass gerechtfertigt ${ }^{34}$. Sollte die Reform Kirchheims missglücken, weil der Konvent sich gegen die observanten Schwestern stellte, so drohte den Schwestern, ähnlich wie den Schwestern

30 Vgl. Winston-Allen, Convent Chronicles (wie Anm. 23) S. 107. Sicher ist nach der Beschreibung von Magdalena Kremer und der Chronik des Klosters Schönensteinbach, dass Barbara Bernheimer ursprünglich aus Kirchheim stammte und dann in das observante Kloster in Silo ging, um die Regeln der Reform kennenzulernen und danach zu leben. Als es dann um die Reform des Klosters Kirchheim ging, wurde sie zu dessen Äbtissin ernannt, was ihr ermöglichte, in ihre alte Heimat zurückzukehren.

31 Vgl. Anhang.

32 Ebd.

33 Ebd.

${ }^{34}$ Vgl. Arnold Angenendt: Geschichte der Religiosität im Mittelalter. Darmstadt ${ }^{4} 2009$. S. 509. Während der Belagerung der Kirchheimer Bürger durch den Konflikt des Klosters mit Eberhard dem Jüngeren empfingen die Schwestern regelmäßig die Kommunion, gleichsam als Schutz vor den kommenden Gefahren und Versicherung, dass Gott bei ihnen ist. Gleichzeitig konnte dies dazu dienen, die äußere Bedrohung und die existentielle Not der Schwestern sichtbar zu machen. Vgl. Kirchheimer Chronik (wie Anm. 1) S. 177. 
aus Liebenau in Worms und Coeli Corona in Hochheim ${ }^{35}$, dass sie keinen Zufluchtsort mit Klausur mehr hätten und von Kloster zu Kloster irren müssten, bis sich auf Geheiß der Ordensoberen ein anderes Kloster für die Reform gefunden hätte. Die „Wegzehrung“ sollte auch der Angst vor der Fremde, der anderen Region, vor dem fremden Dialekt, den Gewohnheiten und Traditionen des neuen Klosters entgegensteuern, weil die Schwestern in und aus der Gnade Gottes handelten.

Zur standesgemäßen Durchführung der Reform waren aufwändige Vorbereitungen nötig. Dafür war Johannes Pruser, der Ordensprovinzial und Prior des Stuttgarter Dominikanerklosters, bei den Grafen von Württemberg unterwegs, um für die Transportmittel und sicheres Geleit zu sorgen: Also bracht er in x tagen mit im wider gan Stroßburg und gan Sletzstatt erliche und sycher geleytschafft mit verhengten hangenen wegen, der warent iii die besten wegen, so unser gnedigen frowen von Würtenberg bettent, die schyckenten sy von lieb mit güten furknechten und giten rossen disen swestern ... ${ }^{36}$.

Bemerkenswerterweise werden hier auch die Transportmittel für die Schwestern genauer beschrieben: Die drei Wagen, die offenbar von den württembergischen Fürstinnen ${ }^{37}$ Margarethe von Savoyen ${ }^{38}$ (1420-1479) und Elisabeth von Brandenburg $^{39}$ (1451-1524) - Gemahlin bzw. Schwiegertochter Graf Ulrichs V. - zur Verfügung gestellt wurden, waren also gut geschützt und verhangen. Darin zeigt sich die Sorge des Landesherren für die Sicherheit und die Aufrechterhaltung der Klausur und der Consuetudines des Klosters Silo für die reisenden Schwestern. Die Wagen sollten, anders als bei normalen Reisenden, keinen Blick nach außen frei geben, sondern den Schwestern eine Privatsphäre für ihre Gebete schaffen, denn auch auf der Reise mussten sie die Klausur wahren, die Chorgebete sprechen und singen. Der Transport war so angelegt, dass die Schwestern möglichst wenig mit der Außenwelt kommunizierten, nicht abgelenkt wurden, sondern sich ganz auf Gott konzentrieren und ihre Seelen rein erhalten konnten. Zu Recht nennt Hamburger diese Art zu reisen „a form of moving enclosure ${ }^{\star 40}$.

35 Besagte Schwestern wollten in Straßburg 1437 das Reuerinnenkloster reformieren, scheiterten jedoch an den Widerständen und mussten in dem Straßburger Kloster St. Nicolaus in Undis aufgenommen werden, bis sie das Kloster St. Katharina in Colmar reformieren konnten. Vgl. dazu Meyer (wie Anm. 11) S. 97-100.

${ }^{36} \mathrm{Vgl}$. Anhang.

37 In der Kirchheimer Chronik werden Margarethe von Savoyen und Elisabeth von Brandenburg auch als Befürworterinnen der Reform angeführt. Vgl. Kirchheimer Chronik (wie Anm. 1) S. 174-175. Allgemeiner dazu die Beiträge in dem Sammelband: Fürstin und Fürst. Familienbeziehungen und Handlungsmöglichkeiten von hochadeligen Frauen im Mittelalter (Mittelalter-Forschungen 15). Hg. von Jörg Rogge. Ostfildern 2004.

${ }^{38}$ Vgl. Fritz (wie Anm. 27).

39 Vgl. Dieter Stievermann: Elisabeth von Brandenburg. In: Haus Württemberg (wie Anm. 27) S. 100.

${ }^{40}$ Hamburger (wie Anm. 1) S. 125. 


\section{Die Stationen der Reise}

Die Siloer Schwestern reisten von Schlettstadt aus in ihren Wagen zunächst am Oberrhein entlang nach Nordosten, über Ettlingen und den Nordschwarzwald nach Pforzheim, von dort aus weiter nach Stuttgart, Weiler und Kirchheim (Abb. 1). Geht man davon aus, dass die Reisegesellschaft auf den damaligen Straßen mit Gefolge und Gepäck zwischen 30 und $45 \mathrm{~km}$ am Tag zurücklegte ${ }^{41}$, waren die Strecken zwischen Schlettstadt und Straßburg, Lichtenau und Ettlingen sowie Pforzheim und Stuttgart lange und anstrengende Etappen ${ }^{42}$.

Die Reise der Dominikanerinnen begann am 6. Mai 1478 und führte von Silo, dem Heimatkloster der Nonnen in Schlettstadt, über das Margarethenkloster in Straßburg, wo weitere Schwestern für die Reform des Klosters Weiler bei Esslingen ausgewählt worden waren und zur Mitreise bereit standen. Vermutlich überquerten sie in Straßburg den Rhein und reisten dann über Lichtenau und Ettlingen nach Pforzheim weiter. Dort feierten sie das Pfingstfest unter besonderer Verehrung des Heiligen Geistes, in dessen Auftrag sie unterwegs waren. Während des dreitägigen Aufenthaltes vom 9. bis 11. Mai 1478 nutzte Magdalena Kremer die Zeit, um zwei Pforzheimer Schwestern Textur und Malen zu lehren. Die nächste Etappe war Stuttgart, wo die Schwestern ihren Provinzial trafen, der sie auf ihrem Weg nach Weiler begleitete, um am Pfingstdienstag das dortige Dominikanerinnenkloster zu reformieren. Die für Kirchheim vorgesehenen Schwestern sollten dann erst am Freitag danach weiterreisen und im Beisein hoher kirchlicher und weltlicher Autoritäten die Reform durchführen ${ }^{43}$.

Betrachten wir die einzelnen Reisestationen genauer, gerade um die Möglichkeiten für Kontakte zu erfahren und die Netzwerke der dominikanischen Reform näher kennenzulernen.

\subsection{Kloster Silo in Schlettstadt}

Schlettstadt, gelegen an der Straßenverbindung zwischen Straßburg und Colmar, war im 15. Jahrhundert eine florierende Reichsstadt ${ }^{44}$. Das Kloster Unserer Lieben Frau der Dominikanerinnen in Silo innerhalb der Stadtmauern wurde um

${ }^{41}$ Vgl. die üblichen Schätzwerte bei Norbert Obler: Reisen im Mittelalter. München 1986. S. 140 .

42 Vgl. Bernhard Neidiger: Das Dominikanerkloster Stuttgart, die Kanoniker vom gemeinsamen Leben in Urach und die Gründung der Universität Tübingen. Konkurrierende Reformansätze in der württembergischen Kirchenpolitik am Ausgang des Mittelalters (Veröffentlichungen des Archivs der Stadt Stuttgart 58). Stuttgart 1993. S. 78.

${ }^{43}$ Kirchheimer Chronik (wie Anm. 1) S. 177.

${ }^{4}$ Vgl. Paul Adam: Der Humanismus zu Schlettstadt. Die Schule, de Humanisten, die Bibliothek. Obernai 1975. S. 74. - Sigrid Metken: Chronik von Schlettstadt. München 1977. S.2-3. - Daneben auch Karlheinz Ebert: Das Elsaß. Wegzeichen europäischer Geschichte und Kultur zwischen Oberrhein und Vogesen. Köln 1982. S. 184-185. 


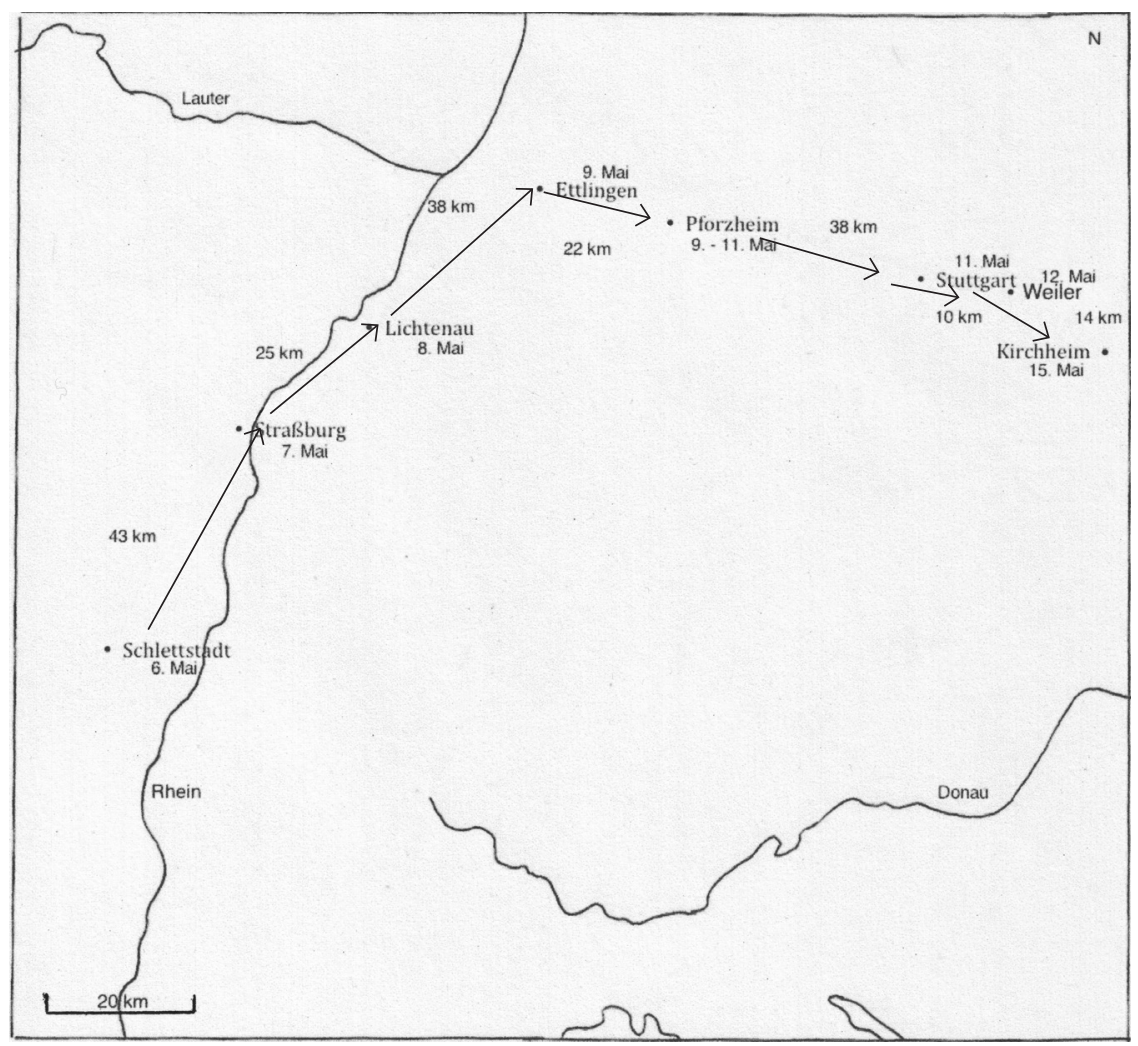

Abb. 1: Reiseweg der Schwestern von Schlettstadt nach Kirchheim unter Teck 1478.

1258 gegründet. Die Schwestern waren ursprünglich laut Johannes Meyer ${ }^{45}$ aus St. Nikolaus bei Rappertsweiler gekommen, von wo sie wegen Unstimmigkeiten mit der Gründerfamilie hatten weichen müssen ${ }^{46}$. Bereits 1464 wurde das Kloster mit Hilfe des Stadtrates und einiger observanter Dominikaner reformiert ${ }^{47}$. Offenbar baten die Schwestern selbst mit Unterstützung des Rates der Stadt um die Einführung der Observanz. Fünf Schwestern aus dem Katharinenkloster in Colmar reis-

45 Vgl. Meyer (wie Anm. 11). S. 112-113.

46 Vgl. Guy Sichler: Sélestat, douze siècles d'histoire. In: Sélestat. Hg. Jacques Almira u. a. Strasbourg 2000. S. 29- 38. Hier: S. 30.

${ }^{47} \mathrm{Vgl}$. Annette Barthelmé: La réforme dominicaine au XVe siècle en Alsace et dans l'ensemble de la provence de Tuetonie. Strasburg 1931. S 82. 
ten daher nach Silo ${ }^{48}$, wie die Priorin des Klosters in Colmar beschreibt, um die Reform durchzuführen: ... Und diese schwestern empfiengen alle morgens am Montag ehe sie verreisten das Hl. Sakrament und fierte sie der Vicarius mit sich binweg geben Schlettstadt und ritten ibnen entgegen die stiffteren und vill berren von Schlettstadt mebr dan ein meilwegs und geleitheten sie mit grosser ebrerbietsamkeit bis geben Schlettstadt ... ${ }^{49}$

Die Parallelen zu der Reise der Siloer Schwestern nach Kirchheim sind leicht greifbar: An erster Stelle steht das Zusammenwirken des Ordens bzw. des Vikarius, der Stifter und Stadträte von Schlettstadt um die Reform im Kloster einzuführen. Nach längerem Briefwechsel zwischen Colmar und Silo gelingt es, Schwestern für die Reform in Silo zu finden. Auch diese Colmarer Schwestern empfangen noch das heilige Sakrament vor ihrer Reise ${ }^{50}$. Und nicht zuletzt ist die große Ehrerbietung, die der Reform und ihren Trägerinnen entgegen gebracht wird, vergleichbar.

\subsection{Straßburg}

Die Siloer Schwestern verbrachten eine Nacht im Margarethenkloster in Straßburg und nahmen von dort Schwestern mit, die Kloster Weiler bei Esslingen reformieren sollten. 1242 von den Rittern von Girbaden gegründet, wurde das Margarethenkloster vor 1258 nach Straßburg verlegt und zählte zu den reicheren Klöstern mit Besitz meist außerhalb der Stadt ${ }^{51}$. Die Einführung der Observanz erfolgte durch die Aufnahme von Schwestern des reformierten St. Agnes Klosters in Straßburg, das wegen eines Heereszugs Karls des Kühnen 1475 abgerissen worden war. Offensichtlich gab es Kräfte, die die Reform des Margarethenklosters befürworteten, denn die führenden Ämter im Kloster wurden mit reformierten Nonnen aus St. Agnes besetzt, woraufhin die ehemalige Priorin und einige Mitschwestern das Kloster verließen. Erst $1476 \mathrm{kam}$ es zu einem Ausgleich aus dem Klostervermögen für die ausgetretenen Schwestern ${ }^{52}$.

Das St. Margarethenkloster war zum Zeitpunkt der Reise der Siloer Nonnen fest in der vielfältigen Klosterlandschaft Straßburgs etabliert und einer der Schwerpunkte der Observanz im Elsass. Zur Zeit der Reise im Jahr 1478 war das Kloster also im Stande und bereit, Schwestern für die Reform nach Württemberg zu schicken.

${ }^{48}$ Vgl. Meyer (wie Anm. 11). S. 112.

49 Ebd. S. 114.

50 Die Kirchheimer Chronik spricht von einer vollen Absolution aller ihrer Sünden, die den Schwestern vor der Reise neben dem Sakrament von Johannes Meyer erteilt worden sei; Kirchheimer Chronik (wie Anm. 1) S. 176.

51 Vgl. dazu Hirbodian (wie Anm. 11). - Sowie demnächst Dies.: Geistliche Frauen und städtische Welt. Kanonissen, Nonnen und Beginen und ihre Umwelt am Beispiel der Stadt Straßburg im Spätmittelalter (1250-1525). Habilitationsschrift Mainz 2001 (im Druck).

52 Vgl. Hirbodian (wie Anm. 51). 


\subsection{Lichtenau und Ettlingen}

Die Reise war offenbar so geplant, dass die observanten Schwestern jeweils möglichst auch in observanten Dominikanerinnenklöstern übernachten konnten, was die Kosten der Reise und die Gefahren verminderte, die Kontinuität des geistlichen Lebens aber stärkte. In Lichtenau und Ettlingen, zwei Amtsstädten der Markgrafen von Baden, boten sich allerdings keine Klöster des Dominikanerordens an, so dass man dort für eine andersartige Unterbringung sorgen musste. Magdalena Kremer verliert dazu kein Wort.

\subsection{Pforzheim}

Die nächste in der Chronik genannte Station der reisenden Schwestern war Pforzheim, wo ein längerer Aufenthalt eingeplant war. Hier feierten sie das Pfingstfest. In Pforzheim hatten die Dominikaner 1279 mit Erlaubnis des Markgrafen Hermann VII. von Baden ein Kloster im östlichen Teil der neuen Stadt errichtet. Ihr spiritueller Einfluss bewirkte, dass ein im Jahr 1257 gegründetes Reuerinnenkloster 1287 zur Regel der Dominikanerinnen übertrat. Im Jahr 1478, als die Siloer Schwestern nach Pforzheim kamen, war dieses Kloster nach einem Brand mit Hilfe des Landesherren, des Markgrafen von Baden, neu errichtet worden ${ }^{53}$. Der Dominikanerinnenkonvent in Pforzheim setzte sich zu diesem Zeitpunkt meist aus adeligen Frauen zusammen und war mit der Pforzheimer Ober- und Bürgerschicht eng verbunden ${ }^{54}$.

Wie die Chronik des Klosters Schönensteinbach ausführt, war das Pforzheimer Kloster unter Mithilfe des Markgrafen von Baden 1442 von Nürnberg aus reformiert worden. Obwohl die Reform der 26 Pforzheimer Schwestern „wider Willen “55 stattfand, gab es keine größeren Probleme. Im Jahr 1478 präsentierte sich das Kloster in Pforzheim als ein observantes, regeltreues Kloster, wo die reisenden Schwestern freudig begrüßt wurden, und man ihnen mit großer Offenheit entgegen kam, wie Magdalena Kremer berichtet, die hier zwei Schwestern ihre Schreibund Malkünste lehrte ${ }^{56}$.

\subsection{Stuttgart}

Von Pforzheim brachen die Dominikanerinnen nach Stuttgart auf, wo Graf Ulrich von Württemberg als Landesherr residierte, der die Reform der Klöster Weiler

${ }^{53}$ Vgl. Stefan Pätzold: Kleine Geschichte der Stadt Pforzheim. Leinfelden-Echterdingen 2007. S. 54.

54 Vgl. Peter Albert: Johannes Meyer, ein oberdeutscher Chronist des 15. Jahrhunderts. In: ZGO 52 (1898) S. 255-263. Hier: S. 255.

${ }_{55}$ Vgl. Meyer (wie Anm. 11) S. 102.

56 Vgl. Anhang. 
und Kirchheim angeregt hatte. Wo genau sich die Dominikanerinnen aus Silo und Straßburg in Stuttgart aufgehalten haben, verrät uns die Kirchheimer Chronik nicht, vielleicht am Grafenhof selbst, wenn sie nicht in Quartieren des Dominikanerklosters in Stuttgart unterkamen.

Graf Ulrich von Württemberg hatte die Niederlassung der observanten Dominikaner gegründet und großen Wert auf die Einhaltung von Armut, Seelsorge und Bildung gelegt. So mischte sich sein Reformwille mit Frömmigkeit und der Fürsorge für seine Residenzstadt. 1474/75, also kurz vor der Reise der Dominikanerinnen, hatte der Ordensprovinzial Johannes Pruser das Amt des Priors in Stuttgart inne, und er war es auch, der sich für die Durchsetzung der Reform in den Klöstern Kirchheim und Weiler einsetzte und sie vorbereitete ${ }^{57}$. Dieser kurze Aufenthalt in Stuttgart ist von besonderer von Bedeutung, da er die Nähe und Unterstützung des Landesherren betont, genauso wie die Befürwortung der Ordensoberen, vor allem des Provinzials, den die Nonnen in Stuttgart trafen, um mit den weltlichen und geistlichen Obrigkeiten gemeinsam die letzte Strecke des Weges zu reisen.

\subsection{Kloster Weiler}

Die erste Station dieser letzten Wegstrecke war das Kloster Weiler bei Esslingen, das unter einer neuen Leitung aus Straßburg reformiert werden sollte. An dem feierlichen Akt nahmen auch die für Kirchheim vorgesehenen Schwestern teil.

Das Dominikanerinnenkloster in Weiler bei Esslingen wurde 1230 erstmals urkundlich erwähnt ${ }^{58}$. Bekannt ist, dass dieses Kloster immer wieder in finanzielle Schwierigkeiten geriet, obwohl das Männerkloster in Esslingen für das geistliche und finanzielle Wohl der Nonnen sorgte ${ }^{59}$. Wie schon für die Reform des Dominikanerklosters 1476, beschloss der Rat auf Wunsch des Landesherren Ulrich von Württemberg 1477, den observanten Provinzial Jakob von Stubach um Hilfe bei der Reform des Dominikanerinnenklosters zu bitten. Nachdruck verlieh dem Reformanliegen sicherlich auch die Tatsache, dass Ulrich nicht mehr bereit war, nicht observanten Bettelorden Almosen und Stiftungen zukommen zu lassen ${ }^{60}$. Nach einem relativ kurzen Vorlauf wurde die Reform dann 1478 mit der Reise der Siloer und Straßburger Schwestern in die Tat umgesetzt.

57 Vgl. Neidiger (wie Anm. 42) S. 26-33. Der Konvent war mit seinen 14 Mitgliedern zu Anfang recht klein und darauf ausgerichtet, Ordensbrüdern die Möglichkeit zu geben, die „artes liberales“ zu studieren, um dann ihre Kenntnisse in der Seelsorge zu erweitern.

58 Vgl. Susanne Uhrle: Das Dominikanerinnenkloster Weiler bei Esslingen (12301571/92). Stuttgart 1969. S. 5.

59 Vgl. Janina Rhein: Dominikanerinnenkloster Weiler. In: Zwischen Himmel und Erde. Klöster und Pflegehöfe in Esslingen. Hg. von Kirsten Fast und Joachim Halbekann. Esslingen 2010. S. 295-297. Hier: S. 295.

60 Vgl. Jörg Bauer: Das Esslinger Dominikanerkloster im 15. Jahrhundert. In: Himmel (wie Anm. 59) S.168-175. Hier: S. 169. 
Magdalena Kremer beschreibt dazu, dass neben einer großen Menschenmenge besunder doctor Wernhervon Stìckarten ${ }^{61}$ sowie weitere Räte Graf Ulrichs bei dem offiziellen Reformakt anwesend waren ${ }^{62}$. Des weiteren berichtet sie, dass die zehn Reformschwestern aus St. Margarethen in Straßburg alle wichtigen Ämter neu besetzten: Priorin wurde Margaretha Hummel von Staufenberg, das Amt der Subpriorin übernahm Beatrix von Mühlheim, das der Schaffnerin Ursula von Colmar, Kusterin und Raderin wurden Magdalena Foelck und Anna Stuentz ${ }^{63}$. Die Chronistin schreibt mit Genugtuung, dass die Zahl der Schwestern nach der Reform in Weiler sprunghaft anstieg ${ }^{64}$.

In Weiler endete die Reise für einen Teil der Reisegesellschaft, und es lässt sich ein gewisses Muster der Reform erkennen: Der Esslinger Frauenkonvent war vermutlich größer als der Kirchheimer, weil die Zahl der ausgewählten Nonnen aus Straßburg für die höchsten Ämter im neu observanten Kloster mit zehn die der ausgewählten Nonnen aus Schlettstadt mit sieben übertraf. Um ein Kloster erfolgreich und auf Dauer reformieren zu können, brauchte man, wenn man die inneren Widerstände der Nonnen, die den Konvent nicht verlassen wollten, berücksichtigt, etwa ein Fünftel bereits reformierter Klosterfrauen, die fähig waren, führende Positionen im Kloster zu übernehmen, die Idee der Reform erfolgreich zu vermitteln und zu verteidigen. Aus der Darstellung des Werdegangs der leitenden Schwestern für die neuen Klöster geht hervor, dass die Auswahl der Abgesandten gezielt vorgenommen wurde. So war die neue Priorin von Esslingen, Schwester Margareta Hummel von Staufenberg, vorher lange Zeit Schaffnerin in ihrem Straßburger Kloster gewesen.

\subsection{Kirchheim}

Die Reise der Siloer Schwestern endete in Kirchheim, dem Ort, den sie reformieren sollten. Dort beginnt der eigentliche Akt der Reform mit dem Einzug der Schwestern in das Kloster Kirchheim unter Teck. Am Freitag in der Pfingstwoche, dem 15. Mai 1478, zogen die sieben Schwestern unter dem Geleit von Dominikanern, dem Provinzial Jakob von Stubach, Johannes Meyer, Johannes Pruser und Jakob Dienstlin in das Kloster Kirchheim ein. Diese vier Oberen der Dominikaner führten die Reform in Kirchheim von Seiten ihres Ordens durch. Die Schwestern wurden von den Grafen von Württemberg, Graf Eberhard dem Älteren und Graf

61 Werner Wick, württembergischer Rat und damals auch Prediger an der Stuttgarter Stiftskirche. Vgl. Oliver Auge: Stiftsbiographien. Die Kleriker des Stuttgarter Heilig-KreuzStifts (1250-1552) (Schriften zur südwestdeutschen Landeskunde 38). Göppingen 2002. S. $582 \mathrm{ff}$.

$62 \mathrm{Vgl}$. Anhang.

63 Die Schwestern aus Straßburg finden sich auch bei Hirbodian (wie Anm. 51).

64 Kirchheimer Chronik (wie Anm. 1) S. 177. Vgl. dazu auch Uhrle (wie Anm. 58) S.6669. 
Eberhard dem Jüngeren, und den Vertretern der württembergischen Geistlichkeit, Doktor Werner von Stuttgart ${ }^{65}$, Doktor Ludwig, Stiftspropst zu Stuttgart und Kirchheim ${ }^{66}$, dem Prior und Visitator von Güterstein (Gutenstein) ${ }^{67}$ und dem Propst von Denkendorf ${ }^{68}$ empfangen, der die Schwestern einsetzte. Die Grafen und ihre Begleiter sowie die Reformer waren anwesend, um durch ihre Präsenz ihre Zustimmung zur Reform zu geben und eventuelle Schwierigkeiten und Widerstände im Kloster Kirchheim im Keim zu ersticken. Die hohe Geistlichkeit und die Räte bzw. Doktoren kamen aus Stuttgart, da Kirchheim zur Grafschaft Ulrichs V. von Stuttgart gehörte.

Bemerkenswert ist, dass der Propst von Denkendorf als der ranghöchste württembergische Geistliche derjenige war, der die Schwestern segnete. Das Chorherrenstift vom Heiligen Grab stand unter der Vogtei der Württemberger Grafen, war aber 1387 in das Esslinger Bürgerrecht aufgenommen und erst 1424 „unter den Schirm und Schutz Württembergs ${ }^{\text {"69 }}$ zurückgekehrt. Eine Beschreibung der Situation im Kloster, die einen Grund für die Reform gegeben hätte, fehlt an dieser Stelle.

Magdalena Kremer beschreibt, dass der alte Konvent von Kirchheim während des Einzugs das „Veni Creator" fröhlich mitsang ${ }^{70}$. Damit erteilte er der Reform seine Zustimmung und unterwarf sich dem Willen des Grafen als Landesherren, der vier anwesenden hochrangigen Dominikaner und der hohen Geistlichkeit. Joachim Homeyer vermutet in seiner Beschreibung der Reform des Zisterzienserinnenklosters Medingen 1479, heute ein Ortsteil von Bad Bevensen in Niedersachsen, dass die Schwestern des alten Konvents vor dem Einzug der Reformierten 14 Tage lang in individuellen Gesprächen überredet wurden, sich zu der Reform bereit zu erklären ${ }^{71}$. Dies könnte auch in Kirchheim der Fall gewesen sein, da die Ordensoberen des observanten Zweiges der Dominikaner bei dem Einzug der Siloer Schwestern bereits anwesend waren und auch die Kirchheimer Schwestern mit ihrem Einzugslied „Veni Creator“ signalisierten, dass sie über die bevorstehende Reform informiert waren.

${ }_{65}$ Vgl. Ange (wie Anm. 61).

66 Ebd. S. $508 \mathrm{f}$.

${ }^{67}$ Vgl. Roland Deigendesch: Die Kartause von Güterstein. Geschichte, geistiges Leben und persönliches Umfeld (Schriften zur südwestdeutschen Landeskunde 39). Leinfelden-Echterdingen 2001. S. 275.

68 Vgl. Kaspar Elm: St. Pelagius in Denkendorf. Die älteste deutsche Propstei des Kapitels vom Heiligen Grab in Geschichte und Geschichtsschreibung. In: Landesgeschichte und Geistesgeschichte. Festschrift für Otto Herding zum 65. Geburtstag. Hg. von Kaspar Elm u. a. Stuttgart 1977. S. 80-131. Hier: S. 93.

${ }^{69} \mathrm{Vgl}$. Andrea Denke: Denkendorf. In: Württembergisches Klosterbuch. Klöster und Stifte von den Anfängen bis zur Gegenwart. Hg. von Wolfgang Zimmermann und Nicole Priesching. Sigmaringen 2003. S. 211.

70 Kirchheimer Chronik (wie Anm. 1) S. 178.

71 Vgl. Johannes Homeyer: 500 Jahre Äbtissinnen in Medingen. Uelzen 1994. S. 29. 
Der eigentliche Vollzug der Reform ist der Autorin weniger wichtig als die Neubesetzung der Ämter und die Charakterisierung der neuen Amtsinhaberinnen ${ }^{72}$ : Der Einzug in das Kloster Kirchheim endet im Kapitelsaal, in dem unter dem Vorsitz des Provinzials Jakob von Stubach im Beisein der weltlichen und geistigen Würdenträger alle ding ordentlich vollbracht als dann dazu gebört ${ }^{73}$.

Eine Charakterisierung der neuen Schwestern zeigt, welche Fähigkeiten und Haltungen maßgebend für das Gelingen der Observanz waren und lädt den Leser oder Zuhörer zum Nachahmen ein. Magdalena Kremer beschreibt sich und ihre Mitschwestern als dem Orden zum Teil schon von Kindesbeinen an zugehörig, als Teilnehmer der Siloer Reform und zum Teil auch aus anderen Klöstern stammend. Wichtige Charakterzüge dieser Nonnen waren in ihrer Selbstdarstellung Intelligenz, Geschick, Gewandtheit in geistlichen und weltlichen Dingen, Ehrsamkeit, Freundlichkeit und Andacht ${ }^{74}$. Alle diese Schwestern hatten eine grundsätzliche Gewissensentscheidung, conscientz ${ }^{75}$, für die Reform getroffen. Damit übertrafen die Siloer Nonnen die gewöhnlichen Klosterfrauen, die Gottes- und Menschenliebe hatten, fromm und gehorsam sein sollten. Die Nonnen der Reform wurden wegen ihrer Eigenschaften ausgewählt, weil sowohl von geistlicher als auch von weltlicher Seite Widerstände im Kloster vorausgesehen wurden.

Allerdings gab es in Kirchheim keinen offenen Widerstand gegen die Reform, denn Schwestern, die mit der Reform nicht einverstanden waren, bekamen die Erlaubnis, sich ein anderes Kloster zu suchen. Das verminderte das Unzufriedenheitspotential in dem neu reformierten Kloster. Und tatsächlich nahmen die Schwestern Barbara Schilling, Margareta Rechner, Anna Dürr und Anna Küntzl die letzte Gelegenheit wahr und verließen den Kirchheimer Konvent ${ }^{76}$. Von diesen Schwestern kehrten allerdings drei bald zurück, Barbara Schilling begab sich in das nicht reformierte Kloster in Esslingen Sirnau ${ }^{77}$.

\section{Kulturtransfer der Observanz}

In der Kirchheimer Chronik gilt das observante Dominikanerinnenkloster Pforzheim als das einzige schon reformierte Kloster, das nicht direkt für die Reform der Klöster Weiler oder Kirchheim von Nutzen war. Während Silo und Straßburg benötigt wurden, um Schwestern für die Reform zu rekrutieren, bildete Pforzheim eine Ausnahme.

\footnotetext{
72 Vgl. Kirchheimer Chronik (wie Anm. 1) S. 178-179.

73 Ebd.

74 Ebd.

75 Ebd.

76 Ebd. S. 179.

77 Ebd.
} 
Betrachtet man den Reiseverlauf, so sticht der lange Aufenthalt in Pforzheim mit der Feier des Pfingstfestes hervor. Die Reise fand sicherlich sowohl wegen des guten Wetters zu dieser Zeit des Jahres statt, als auch wegen der liturgischen Bedeutung Pfingstens. Die Ordensprovinzialen lassen die Siloer Schwestern in der Pfingstwoche in Kirchheim einziehen, während die Kirchheimer Schwestern „veni creator" singen, das einerseits ein hohes Pfingstlied ist, andererseits aber auch ihre Akzeptanz für die Reform ausdrückt: Der Herr möge kommen um die Reform und mit ihr die observanten Schwestern zu bringen, und nur mit der Hilfe des Dreieinigen Gottes, besonders des Heiligen Geistes, kann die Reform gelingen.

Bedenkt man diese Symbolik des Einzugs der Siloer Schwestern in Kirchheim, so drängt sich der Schluss auf, dass auch der lange Aufenthalt über Pfingsten in Pforzheim entsprechend arrangiert gewesen sein musste. Pforzheim, das vom Katharinenkloster in Nürnberg ${ }^{78} 1442$ reformiert worden war, schien zu diesem Zeitpunkt nicht genügend qualifizierte Schwestern im Kloster zu haben, um die Reform Kirchheims oder Weilers zu unterstützen. Der Grund dafür dürfte die Reform 1467 St. Marias in Medlingen bei Dillingen gewesen sein, wohin vier Schwestern von Pforzheim geschickt worden waren ${ }^{79}$. Erstaunlich ist, dass Pforzheim nicht von Nürnberg aus weiter unterstützt wurde. Dies zeugt gleichzeitig von der hohen Bildung und Ausstattung der Nonnen aus Schlettstadt. Magdalena Kremer scheint eine Künstlerin mit besonderen Gaben gewesen zu sein, die wohl auch den Ordensprovinzialen aufgefallen war, denn sie ist diejenige, die die Pforzheimer Schwestern auf ihrem Missionsweg der Reform weiterbildete. Dieser Austausch von Wissen war ganz im Sinne der Observanz und wurde sicherlich von den Ordensoberen auf jede Weise gefördert, um für die nötige Bildung im Kloster in Pforzheim zu sorgen.

Mit dieser Belehrung über die Textur und das Malen von mehreren Schwestern sicherten die Dominikaner auch eine wichtige finanzielle Grundlage des Klosters. Mit einer gut angesehenen Schreibstube konnte man im Kloster Schreibaufträge vom Orden selber, aber auch von Laien, Adeligen und Bürgern annehmen. Gleichzeitig konnte das Kloster in Pforzheim auf diese Weise trotz der strengen Klausur der Schwestern am Diskurs über die Reform innerhalb des Ordens teilnehmen. Es war damit nicht nur empfangendes Kloster von Schriften der Observanz, sondern konnte sich auch aktiv an deren Diskussion und Gestaltung beteiligen.

Jeffrey Hamburger sieht in seinem Aufsatz über die Chronistin Magdalena Kremer $^{80}$ eine enge Verbindung der elsässischen Klöster Straßburg, Colmar und

78 Vgl. Barbara Steinke: Paradiesgarten oder Gefängnis? Das Nürnberger Katharinenkloster zwischen Klosterreform und Reformation (Spätmittelalter und Reformation. Neue Reihe 30). Tübingen 2006. - Antje Willing: Literatur und Ordensreform im 15. Jahrhundert. Deutsche Abendmahlsschriften im Nürnberger Katharinenkloster (Studien und Texte zum Mittelalter und zur frühen Neuzeit 4). Münster u. a. 2004.

79 Vgl. Barthelmé (wie Anm. 47) S. 67.

80 Vgl. Hamburger (wie Anm. 1) S. 124-149. 
Schlettstadt, die er unter anderem an ihrem ähnlichen Stil in der Gestaltung der Nekrologe und liturgischen Manuskripte festmacht. Für ihn sind auch die Kirchheimer Handschriften Importe aus dem Elsass, die die Wissensgemeinschaft und Weitergabe der Reform zum Ausdruck bringen. Damit kann man in der Reform der observanten Klöster gleichzeitig eine Kulturreform erkennen, denn die elsässischen Klöster gaben in der Person ihrer mitgeschickten ausgebildeten Schreiberinnen ihre Art der Gestaltung und Schreibfähigkeit weiter. So zeigt sich neben der zentralen theologischen Rolle der Reform im Leben der Schwestern das Programm einer klösterlichen, liturgischen Erneuerung. Die Schwestern zu befähigen, an dem Diskurs um die Reform in Form von Schriften teilzunehmen, gab ihnen eine Stimme, die sie trotz der Klausur in die Lage versetzte, ihre Meinung und ihren Glauben kund zu tun.

Als das Kirchheimer Kloster in äußerster Bedrängnis durch den eigenen Landesherren Eberhard den Jüngeren geraten war, wandte sich die Priorin im Jahr 1490 an weltliche und geistliche Fürsten und Adelige um Hilfe gegen die Belagerung durch den Landesherren Eberhard des Jüngeren: Ist unser Begeren, [...] um bylff, trost und bystand, zu allen Christlichen fürsten, ryttern, edlen und allen anderen um gottes und marie der hymlischen künigin wyllen. Das uns armen wyplichem geschleth. Zu Hylff kumen werde, damit wir nit so unchristlich verderbt werdent, das wollent wir gegen Gott mit unserem Verdienst ewiglich gedenken ${ }^{81}$.

Sie konnte sich mit der Bitte um Gebet an den nächsten Kreis von Dominikanerklöstern in Ulm, Stuttgart, Esslingen, Gmünd und Pforzheim wenden, unter diesen auch all die observanten Dominikanerinnenklöster, die die Siloer Schwestern auf der Reise von Silo nach Kirchheim besucht hatten ${ }^{82}$. Die Reiseetappen der Schwestern entwickelten sich zur Kontaktaufnahme und Weitergabe von Wissen, was in der Stunde der Not für die Bewahrung der Reform durch Gefahr von außen lebensrettend wurde.

Die Reise der Schwestern war zwar keine Pilgerreise zu heiligen Stätten wie Jerusalem, Santiago oder Rom im herkömmlichen Sinn, denn die Schwestern reisten unter Einhaltung der Klausur durch die „moving enclosure“ von Ort zu Ort und kehrten nie in das observante Kloster zurück, von dem sie aufgebrochen waren, aber es handelt sich hierbei dennoch um eine Reise, die vom Frömmigkeitsideal der damaligen Zeit motiviert war. Die Schwestern hatten sich mit ihrem Entschluss der Weitergabe der Lehre und der Ideale der Observanz für eine Form des existenziellen Reisens entschieden und waren entschlossen, ihre neue Art der Frömmigkeit in der Fremde zu leben und anderen den idealen Weg zu Gott zeigen.

Der Weg der Schwestern von Silo nach Kirchheim war vom Landesherren und den Ordensprovinzialen bis ins Detail geplant und mit ausreichend Geld und Mitteln unterstützt worden. Die Hilfe der Grafen von Württemberg bei dieser auf-

${ }^{81}$ Kirchheimer Chronik (wie Anm. 1) S. 275-276.

82 Ebd. S. 276. 
wändigen Reise lässt auch in den nachfolgenden Ereignissen auf die beständige Unterstützung Graf Eberhards des Älteren für das Kloster Kirchheim gegen seinen Vetter Eberhard den Jüngeren verweisen.

\section{Fazit}

Lässt man zum Abschluss die Reise der Siloer Schwestern noch einmal Revue passieren, ergeben sich wichtige Aufschlüsse in Hinblick auf Reisen für die Klosterreform im späten Mittelalter. Zunächst ist die Perspektive der Nonnen einzunehmen, die sich im Gehorsam auf den Weg von ihrer geistigen Heimat, ihrer Ruhe in Gottesdienst und Gebet sowie der „stabilitas loci“ wegbegeben, hin zu Problemen, Neuem - und das zum Teil in fortgeschrittenem Alter -, um den Willen Gottes zu erfüllen. Sie sind ausgewählt aus den anderen Klöstern und Schwestern, abgesondert von der Welt und damit besonders geheiligt. Andererseits war die Reise öffentlich, gut geplant und durchgeführt, mit Rücksichtnahme auf die Klausur und die Gebete, in kurzen Etappen und Stationen in Klöstern der Reform zum Ausruhen und Lehren. Die Normalität des klösterlichen Alltags wurde soweit möglich beibehalten und von der Obrigkeit und den Ordensoberen garantiert.

Die Reise selbst erscheint wie eine Transplantation: Das Herz eines Klosters wird durch ein anderes ersetzt, das Leben und die Gewohnheiten dieses neuen Klosters werden verändert, das Kloster erlebt neues Leben, eine neue Blüte und neue Attraktion. Silo hat die Besten der Nachfolgegeneration der Nonnen nach Kirchheim abgegeben, ist als Mutterkloster dadurch zunächst einmal innerlich ärmer geworden.

Der Orden, der den Gehorsam der Schwestern gefordert hat und auch fordern konnte, sieht das übergeordnete Ganze als sein Hauptinteresse. Denn selbstverständlich muss der Orden um den Anforderungen der Theologie und der Lebensgestaltung der Zeit gerecht zu werden, sich zeitgemäß reformieren, zurückbesinnen auf die alten "guten“ Ordnungen und Gewohnheiten. Zugeständnisse an die Reform mussten sowohl die in dem reformierten Kloster lebenden Schwestern als auch die Schwestern aus Silo machen, die noch jährlich von ihrem alten Kloster das Leibgeding ausgezahlt bekommen sollten.

Die Reform des Dominikanerinnenklosters in Kirchheim war ein herrschaftlicher Akt unter Beteiligung von Landesherren und Kirche in den Personen der Grafen und ihrer Räte, der Ordensoberen und der hohen Geistlichkeit Württembergs. Durch deren Anwesenheit wurde der Reformakt auch repräsentativ aufgewertet, reisten doch alle diese Personen offenbar gemeinsam von Stuttgart nach Kirchheim. Hier wurden die neuen Nonnen offiziell in das Kloster und ihre Ämter eingesetzt und als neue Untertanen in Württemberg aufgenommen. Diese Rechtsgeschäfte wurden eingebettet in Liturgie und Gottesdienst, in das Gebet um den Heiligen Geist und das „Veni creator spiritus“, das alte und neue Mitglieder des 
Konvents vereinte. Liturgisches und Formales, Juristisches, Geistliches und Weltliches vereinigten sich in der Umwandlung des Klosters. Gottesdienst, Zeugnis und Dienst wirkten zusammen zum Abschluss einer Mission, in der das zeitgemäße ideale klösterliche Leben für Dominikanerinnen aus dem Elsass nach Württemberg verpflanzt wurde. 


\section{Anhang: \\ Ausschnitt aus der Klosterchronik der Magdalena Kremer}

Der folgend neu edierte Ausschnitt aus der Klosterchronik der Magdalena Kremer von Kirchheim bietet die Reisebeschreibung der Dominikanerinnen von Schlettstadt nach Kirchheim unter Teck. Die Vorlage wurde buchstabengetreu transkribiert mit folgenden Ausnahmen: „u“ und „V“ wurden entsprechend ihres Lautwerts normalisiert; Großschreibung erscheint nur an Satzanfängen und bei Eigennamen. Die Interpunktion wurde in Anlehnung an den heutigen Gebrauch normalisiert. Zusätze des Bearbeiters erscheinen in [], offensichtliche Fehler werden durch ein [!] verdeutlicht.

[1489/90]

Ausfertigung, Papier, mit Rubrizierungen, kleineren Korrekturen und späteren Ergänzungen

Ledereinband, 294 beschriebene Seiten, $21,8 \times 15,5 \mathrm{~cm}$

Hauptstaatsarchiv Stuttgart A 493 Bü 2, S. 10-12 (vgl. Abb. 2)

Edition: Christian Friedrich Sattler: Geschichte des Herzogthums Würtenberg unter der Regierung der Graven. Bd. 4. Tübingen 1768. Beilage 42, S. 176-177 (fehlerhaft)

[S. 10] [...] Also do das volbracht wart, do ritt der vatter Johannes Pruser wider zu der herschafft zu Würtenberg und bracht gute wider botschafft, wie [S. 11] die gute sach so ferre kummen were und das man unverzogenlichen fürbas die sach tryb. Also bracht er in $x$ tagen mit im wider gan Strosburg und gan Sletzstatt erliche und sycher geleytschafft mit verhengten hangenen wegen, der warent iii die besten wegen, so unser gnedigen frowen von Würtenberg bettent, die schyckenten sy von lieb mit güten fürknechten und güten rossen disen swestern, und darzu mit ersamen personen, die sy so ferren wege geleytenten mit kostlicher zerung, das als unser gnediger herr grave Ulrich uß rychtet. Also firt man die zwen wegen gan Sletstatt we und holtent die vii swestern, das sy kement gon Stroßburg zu denen zu sant Margrethen, das sy also mit einander fürent in diß lant. Und also furrent die vii swestern von Syl uß uff Johannis ante portam latinam [1478 Mai 6] am morgen vor tag, und gab jnen ir bychtvatter zi Syl das beylig sacrament zu letz und in das ellend zi eynem geworen geleyt.

Und do sy gan Stroßburg koment, do blybent sy úber nacht zü sant Margrechten und morndes furent sy mit einander, die xvii swestern. Die erste nacht bettent sy herberg zü Liechengnöw, das was uff die oct [ava] ascensionis [1478 Mai 8], die ander nacht zu Ettlingen. [S. 12] Die dritte nacht, dz was derpfyngstaubend, zu Pfortzen, da kament sy uff mittentag in dens das closter, do entpfingent dise xvii bylgerin gar minsamclich und blybent by $\beta$ an den dritten tag. Denn gantzen pfingstag rüwenten sy da und rufftent da mit einander an die mültickeit der gaben des heyl- 
gen geystes. Und ein swester was under den von Syl, die konde wol textur schriben und ouch molen, die lerte zuwo [!] swestern zů Pfortzen die zwen tag.

Am pfingstmentag zugent sy gan Stückart, do kamm zỉ jnen unser wirdiger vatter provincial, und der fir mit jnen gan Wiler uff den pfingstzygstag an sant Nerey et Achylley und Pancracien tag [1478 Mai 12]. Und warent suß vil volckes ouch do und besunder doctor Wernher von Stickarten und andere unsers gnedigen berren retten. Und uff den selben tag wart das closter zu Wiler reformiert in gegenwirtickeit der obgemelten personen und ouch der swestern von Syl, die warent darby und blybent da biß an frytag, do furent sy erst gan Kirchen [...]

Abb. 2 (S. 128-130): Klosterchronik der Magdalena Kremer (HStA Stuttgart A 493 Bü 2, S. 10-12). 


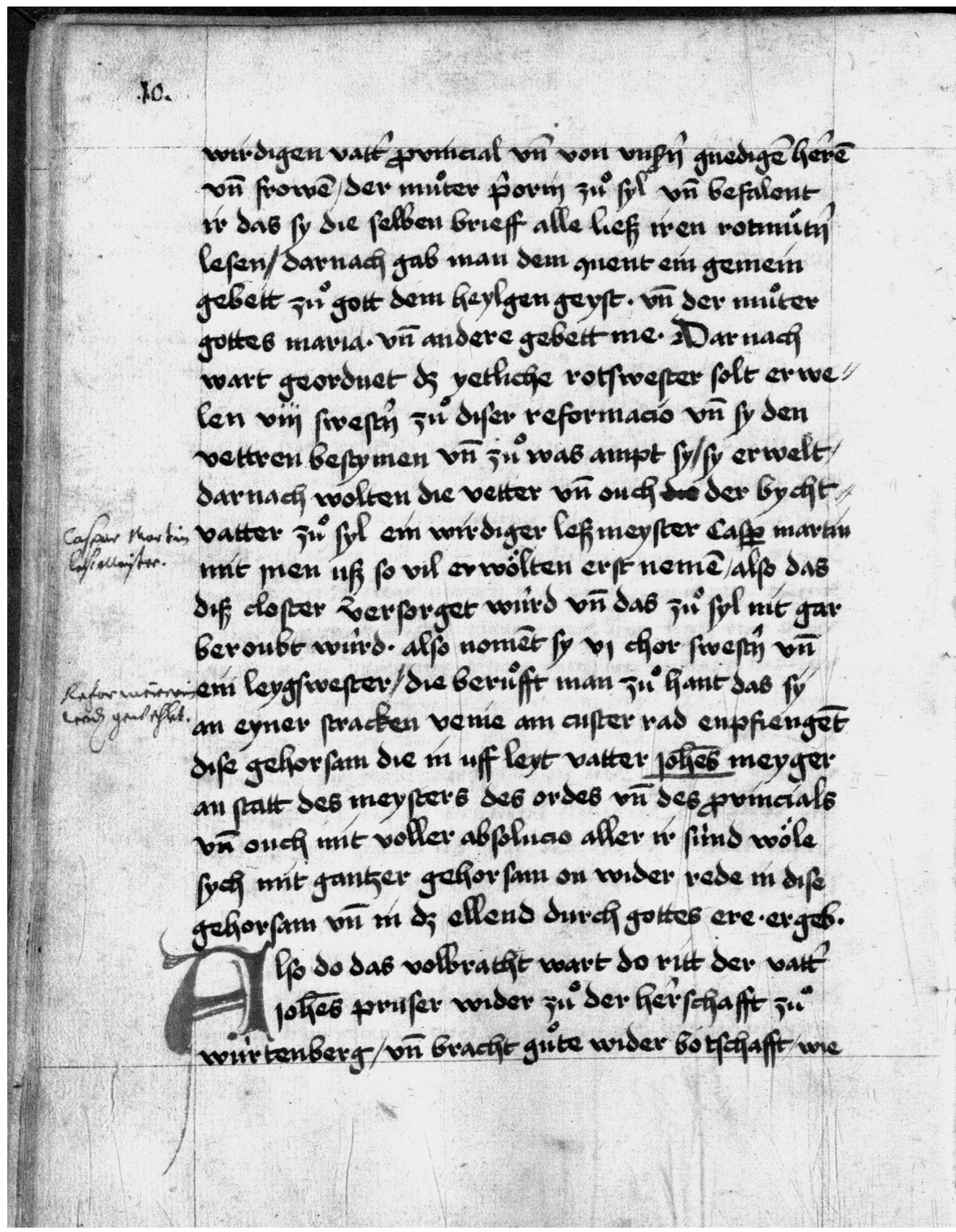


II.

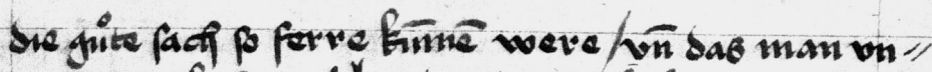

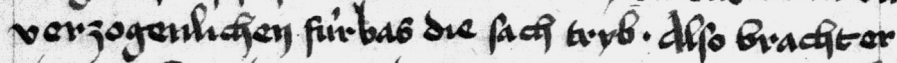

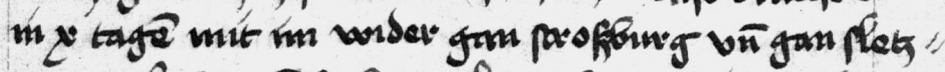

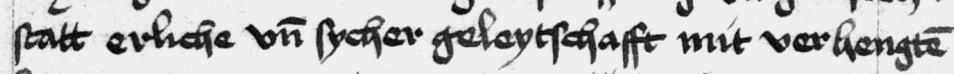
Rangener wegen der warent in she beften weje

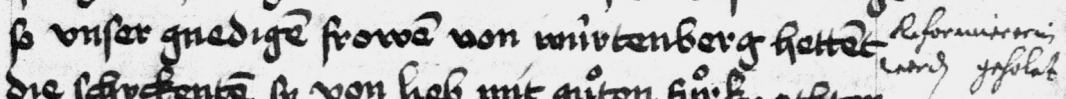
bre pafyente pon hab unt griten fin Enetfitem

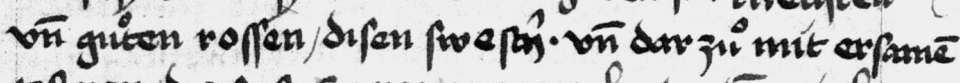
pponen bre 19 p forren wege geleytemte mit fop..

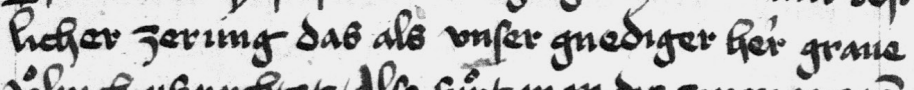

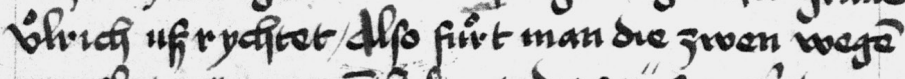
gan pletpatt wh haltent bre on froeph $\$ 46$ (1) Eement gon paofiurg sideney ji pant

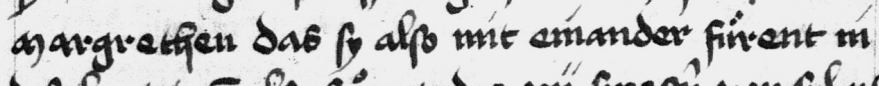
off Pant un alp fitient bue on froeph wen if uf of 1 की ante portan labina am morge vov tag

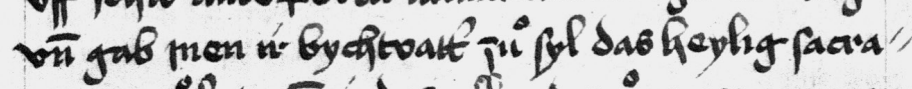

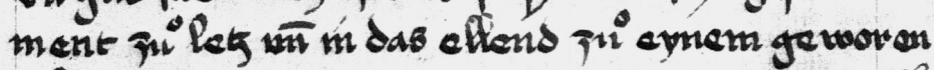
geleyt

it do B gan pooffurug Fomet do blybent iber natit

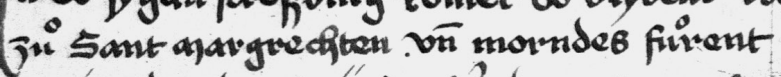

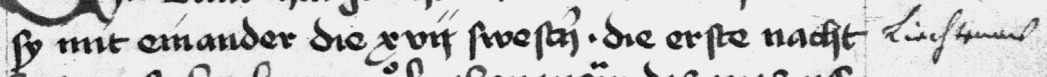
Rettent iy Gerbeng ji hetgengmoio dib nut uff bie oct apentions. bre anber natat jui etthingen. Ethingm. 
12.

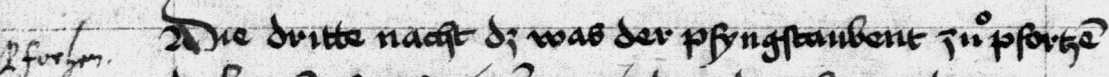
da Eamet iy uff mittétay m dab doper So ent"

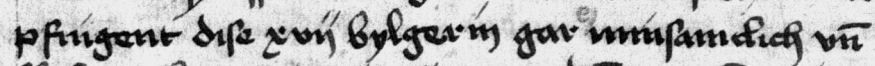

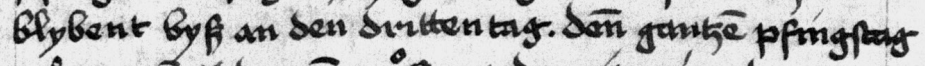
"invente fy da vĩ miftent da mít emátider an bre milticfert der gaben des keylgen geypas. un

ing doma

anderion em fropter was wder den wen ill bie fonde wool

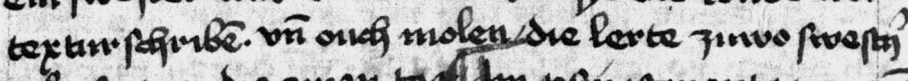

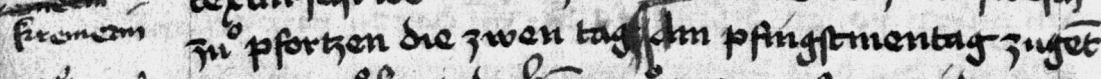

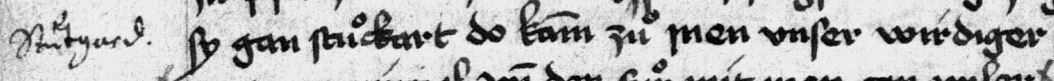
foattev entricial on ber firi unt men gan whent?

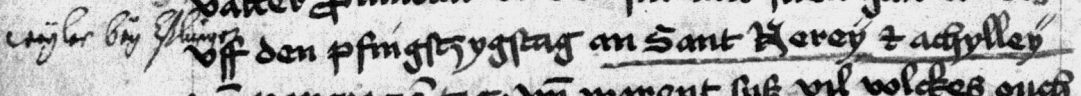

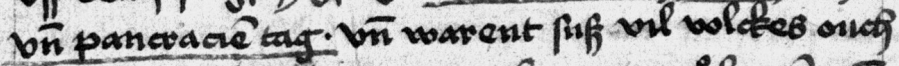

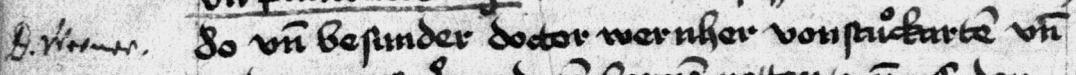
andere unfex'guesuge fowre vetoen/ on uff den

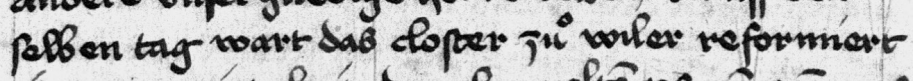

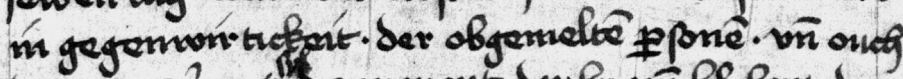

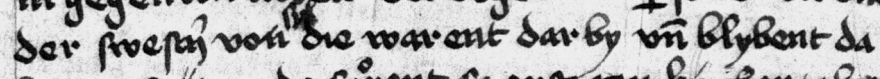

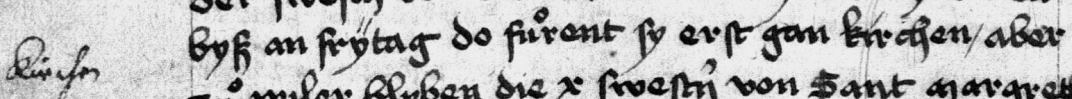

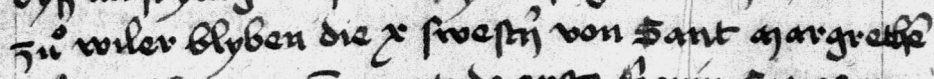

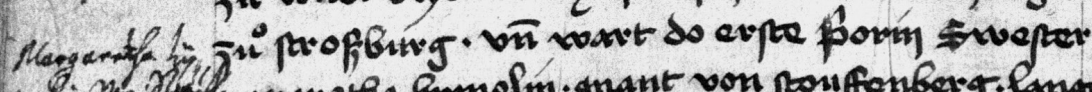

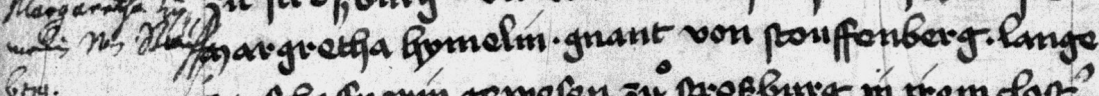

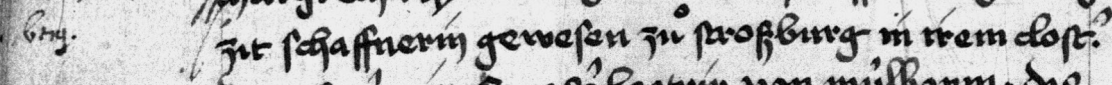

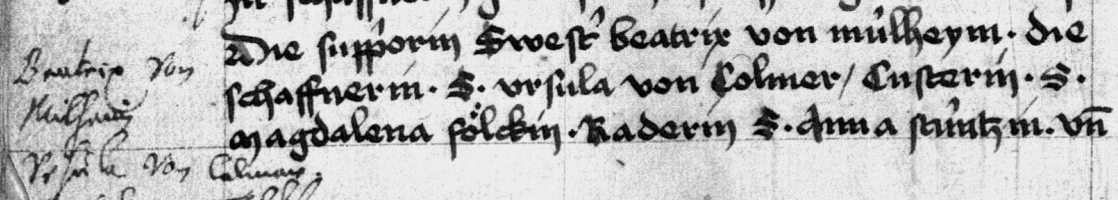

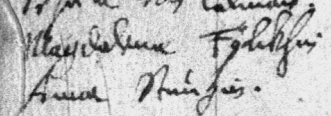

